\title{
ARIA 2019: Um Percurso Assistencial Integrado para a Rinite Alérgica em Portugal
}

\author{
ARIA 2019: An Integrated Care Pathway for Allergic \\ Rhinitis in Portugal
}

\begin{abstract}
João A. FONSECA ${ }^{1,2}$, Tiago TAVEIRA-GOMES ${ }^{2,4,5}$, Ana Margarida PEREIRA ${ }^{1,2,3}$, Manuel BRANCO-FERREIRA ${ }^{6,7,8}$, Pedro CARREIRO-MARTINS ${ }^{9,10}$, Magna ALVES-CORREIA ${ }^{1,2,3}$, Jaime CORREIA DE SOUSA ${ }^{11,12,13}$, Elísio COSTA ${ }^{14}$, Olga LOURENÇO ${ }^{15}$, Mário MORAIS-ALMEIDA ${ }^{16}$, Ana MORÉTE ${ }^{3,17}$, Frederico REGATEIRO ${ }^{18,19,20}$, Ana Todo BOM ${ }^{18}$, Claus BACHERT ${ }^{21}$, Oliver PFAAR ${ }^{22}$, Dana WALLACE ${ }^{23}$, Anna BEDBROOK ${ }^{24}$, Wienczyslawa CZARLEWSKI ${ }^{25}$, Jean BOUSQUET $₫ 24,26,27$

Acta Med Port 2021 Feb;34(2):144-157 • https://doi.org/10.20344/amp.13777
\end{abstract}

\section{RESUMO}

A iniciativa Allergic Rhinitis and Its Impact on Asthma (ARIA) teve início há mais de 20 anos e tem elaborado e disseminado orientações baseadas em evidência, e desenvolvido projetos na área da rinite alérgica. Esta iniciativa está atualmente focada em proporcionar orientações centradas no doente que contribuam para um percurso integrado entre os vários níveis de cuidados e que tirem partido de soluções digitais, tendo sido recomendada a introdução na prática clínica de percursos assistenciais integrados. Neste artigo descrevemos a adaptação para Portugal do documento ARIA Integrated Care Pathways. Após breve revisão sobre a epidemiologia e o impacto da rinite alérgica em Portugal e das atividades realizadas em Portugal no âmbito da iniciativa ARIA, é descrito o conjunto alargado de conhecimento utilizado para o desenvolvimento das recomendações para o tratamento farmacológico da rinite alérgica, recomendações essas baseadas na metodologia GRADE, evidência do mundo real adquirida por tecnologia móvel $(\mathrm{mH} H e a l t h)$ e resultante de estudos de câmara de exposição alergénica. Em seguida, são resumidos os percursos assistenciais integrados para imunoterapia com alergénios produzidas em 2019. Considera-se a imunoterapia com alergénios um exemplo de medicina de precisão e em que a utilização de tecnologias $m$ Health permitirá melhorar a estratificação para seleção dos doentes e monitorização da resposta. Estas recomendações foram consideradas como 'boas práticas' dos cuidados integrados centrados no doente apoiados por sistemas digitais da DG Santé (Direção Geral de Saúde e de Segurança Alimentar da União Europeia) e representam a estratégia de gestão da mudança da fase 4 do ARIA.

Palavras-chave: Asma; Guia de Prática Clínica; Portugal; Prestação Integrada de Cuidados de Saúde; Rinite Alérgica

\section{ABSTRACT}

The Allergic Rhinitis and Its Impact on Asthma (ARIA) initiative started more than 20 years ago and has developed and disseminated evidence-based guidelines and projects in the field of allergic rhinitis. This initiative is currently focused on providing patient-centred guidelines that contribute to an integrated care pathway between the various levels of care and take advantage of digital solutions, and the introduction of integrated care pathways in clinical practice has been recommended. In this article we describe the adaptation for Portugal of the ARIA Integrated Care Pathways document. After a brief review of the epidemiology and impact of allergic rhinitis

1. Centro de Investigação em Tecnologias e Serviços de Saúde (CINTESIS). Faculdade de Medicina. Universidade do Porto. Porto. Portugal.

2. Departamento de Medicina da Comunidade, Informação e Decisão em Saúde (MEDCIDS). Faculdade de Medicina. Universidade do Porto. Porto. Portugal.

3. Serviço de Imunoalergologia. Hospital CUF. Porto. Portugal.

4. Instituto Universitário de Ciências da Saúde. Cooperativa de Ensino Superior Politécnico Universitário. Gandra. Portugal.

5. Faculdade de Ciências da Saúde. Universidade Fernando Pessoa. Porto. Portugal.

6. Clínica Universitária Imunoalergologia. Faculdade de Medicina. Universidade de Lisboa. Lisboa. Portugal.

7. Serviço de Imunoalergologia. Centro Hospitalar Universitário Lisboa Norte. Lisboa. Portugal.

8. Sociedade Portuguesa de Alergologia e Imunologia Clínica. Lisboa. Portugal.

9. Comprehensive Health Research Center (CHRC). NOVA Medical School. Lisboa. Portugal.

10. Serviço de Imunoalergologia. Centro Hospitalar Universitário de Lisboa Central. Lisboa. Portugal.

11. Instituto de Investigação em Ciências da Vida e Saúde (ICVS). Escola de Medicina. Universidade do Minho. Braga. Portugal.

12. Laboratório Associado. Instituto de Investigação em Ciências da Vida e Saúde Braga/Guimarães. Braga. Portugal.

13. Unidade de Saúde Familiar Horizonte. Matosinhos. Portugal.

14. UCIBIO/REQUIMTE. Porto4Ageing Reference Site. Faculdade de Farmácia. Universidade do Porto. Portugal.

15. Faculdade de Ciências da Saúde e Centro de Investigação em Ciências da Saúde. Universidade da Beira Interior. Covilhã. Portugal.

16. Centro de Alergia. Hospital CUF Descobertas. Lisboa. Portugal.

17. Unidade de Imunoalergologia. Centro Hospitalar Baixo Vouga. Aveiro. Portugal.

18. Serviço de Imunoalergologia. Centro Hospitalar e Universitário de Coimbra. Coimbra. Portugal.

19. Instituto de Imunologia. Faculdade de Medicina. Universidade de Coimbra. Coimbra. Portugal.

20. Instituto de Investigação Clínica e Biomédica de Coimbra (ICBR). Faculdade de Medicina. Universidade de Coimbra. Coimbra. Portugal.

21. Upper Airways Research Laboratory. ENT Department. Ghent University Hospital. Ghent. Belgium.

22. Department of Otorhinolaryngology. Head and Neck Surgery. Section of Rhinology and Allergy. University Hospital Marburg. Phillipps-Universität Marburg. Marburg. Germany.

23. Nova Southeastern University. Fort Lauderdale. Florida. United States of America.

24. MACVIA-France. Fondation Partenariale FMC VIA-LR. Centre Hospitalier Arnaud de Villeneuve. Montpellier. France.

25. Mask-Air. Levallois. France.

26. INSERM U 1168. Université Versailles St-Quentin-en-Yvelines. Montigny le Bretonneux. France.

27. Charité. Universitätsmedizin Berlin. Humboldt-Universität zu Berlin. Berlin. Germany.

$\triangle$ Autor correspondente: Jean Bousquet. jean.bousquet@orange.fr

Recebido: 23 de março de 2020 - Aceite: 05 de novembro de 2020 - First published: 04 de dezembro de 2020 - Online issue published: 01 de fevereiro de 2021 Copyright $\odot$ Ordem dos Médicos 2021 
in Portugal and the activities carried out in Portugal within the ARIA initiative, we describe the broad knowledge base used for the development of recommendations for the pharmacological treatment of allergic rhinitis, and these recommendations are based on the GRADE methodology, real world evidence acquired by mobile technology (mHealth) and resulting from allergenic exposure chamber studies. What follows is a summary of integrated care pathways for allergen immunotherapy produced in 2019. Allergen immunotherapy is considered an example of precision medicine where the use of mHealth technologies will improve stratification for patient selection and response monitoring. These recommendations were considered as best practices of integrated patient-centred care supported by digital systems from Directorate General for Health and Food Safety of the European Union (DG Santé) and represent the ARIA Phase 4 Change Management strategy.

Keywords: Asthma; Clinical Practice Guideline; Delivery of Health Care, Integrated; Portugal; Rhinitis, Allergic

\section{INTRODUÇÃO}

Em todas as sociedades, os encargos e os custos das doenças alérgicas estão a aumentar rapidamente, sendo necessárias estratégias de gestão da mudança para apoiar a transformação do sistema de cuidados de saúde em cuidados integrados. ${ }^{1}$ A 3 de dezembro de 2018, realizou-se em Paris uma reunião focada no tratamento de doenças crónicas, organizada pelo Mobile Airways Sentinel Network (MASK) ${ }^{1}$ e pelo Impact of Air Pollution on Asthma and Rhinitis, European Institute of Innovation and Technology Health (POLLAR), ${ }^{2}$ em colaboração com organizações profissionais e de doentes dedicadas a doenças alérgicas e das vias aéreas (Fig. 1). ${ }^{3}$ Tendo por meta a prestação de cuidados integrados, de base digital e centrados na pessoa com multimorbilidade por rinite e asma associada à exposição ambiental, foi recomendada a introdução na prática clínica de percursos de cuidados integrados - integrated care pathways (PAI). ${ }^{1}$

Os PAls são planos de cuidados multidisciplinares estruturados que detalham as principais etapas dos cuidados prestados ao doente. ${ }^{4}$ Promovem a tradução das recomendações clínicas globais em protocolos locais e a sua consequente aplicação na prática clínica. ${ }^{5,6}$ Os PAI airways (PAls para doenças das vias aéreas) ${ }^{5,7}$ constituíram um primeiro passo para o desenvolvimento de PAI para a multimorbilidade por rinite e asma. ${ }^{8,9}$

No que respeita à rinite alérgica $(R A)$, considera-se urgente desenvolver novas orientações para o tratamento farmacológico bem como PAls relativas à imunoterapia com alergénios (ITA). Este documento representa o sumário executivo dessa reunião de Paris (Fig. 2), e a sua adaptação à realidade do sistema de saúde português. Assim, este artigo apresenta o percurso assistencial integrado para a rinite alérgica elaborado em 2019 pela iniciativa ARIA em Portugal, e adaptado à realidade portuguesa.

\section{Situação no país}

\subsection{Prevalência e carga de doença}

Em Portugal, os estudos epidemiológicos sobre rinite estimam uma prevalência de $21,5 \%$ a $24 \%$ em crianças, $27 \%$ em adolescentes, $26,1 \%$ nos adultos e $29,8 \%$ no idoso. ${ }^{10-12} \mathrm{~A}$ maioria dos doentes portugueses reportam sintomas, pelo menos, de gravidade moderada $(52 \%)$, sendo que apenas $30 \%$ tinham um diagnóstico médico, e só um terço $(31,9 \%)$ tinha realizado estudo alergológico com testes cutâneos por picada. ${ }^{13}$

A RA é uma das patologias mais frequentemente auto-diagnosticadas e auto-tratadas, sendo que menos de metade dos doentes procuram aconselhamento ou tratamento médico para os seus sintomas. ${ }^{13}$ Esta atitude resulta num subdiagnóstico ${ }^{11,14,15}$ que leva a um sub-tratamento e a um mau controlo dos sintomas, ${ }^{16-18}$ atingindo fortemente a qualidade de vida dos doentes. ${ }^{19-22} \mathrm{~A}$ maioria dos doentes observados em consulta tem sintomas de RA moderados a graves, e cerca de metade refere aparecimento de sintomas persistentes mais de quatro dias por semana e mais do que quatro semanas consecutivas. ${ }^{19}$

A RA é um problema global de saúde que causa um enorme impacto na qualidade de vida e na produtividade laboral e escolar, ${ }^{23-25}$ pelo que as suas repercussões económicas são potencialmente substanciais. Além disso,

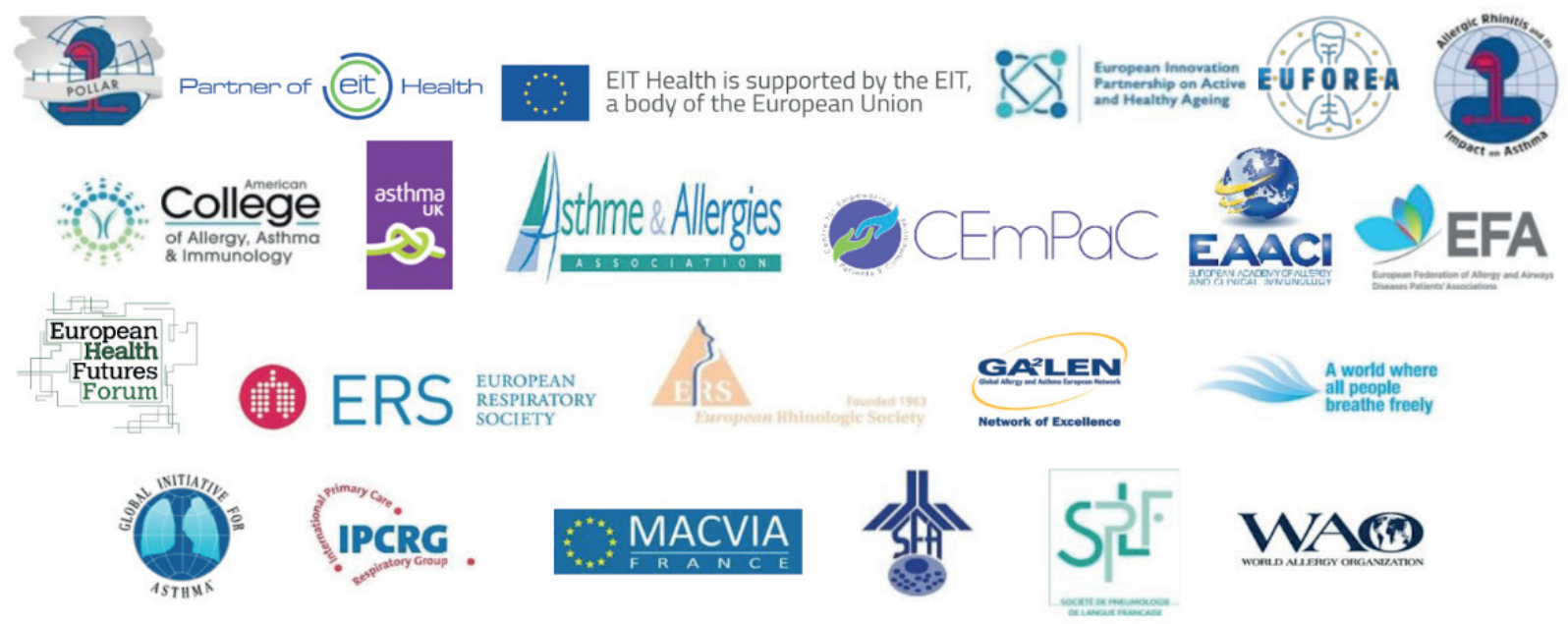

Figura 1 - Organizações que apoiaram a reunião focada no tratamento de doenças crónicas, organizada pela Mobile Airways Sentinel NetworK e pelo Impact of Air Pollution on Asthma and Rhinitis, European Information Technology Health em Paris 2018 


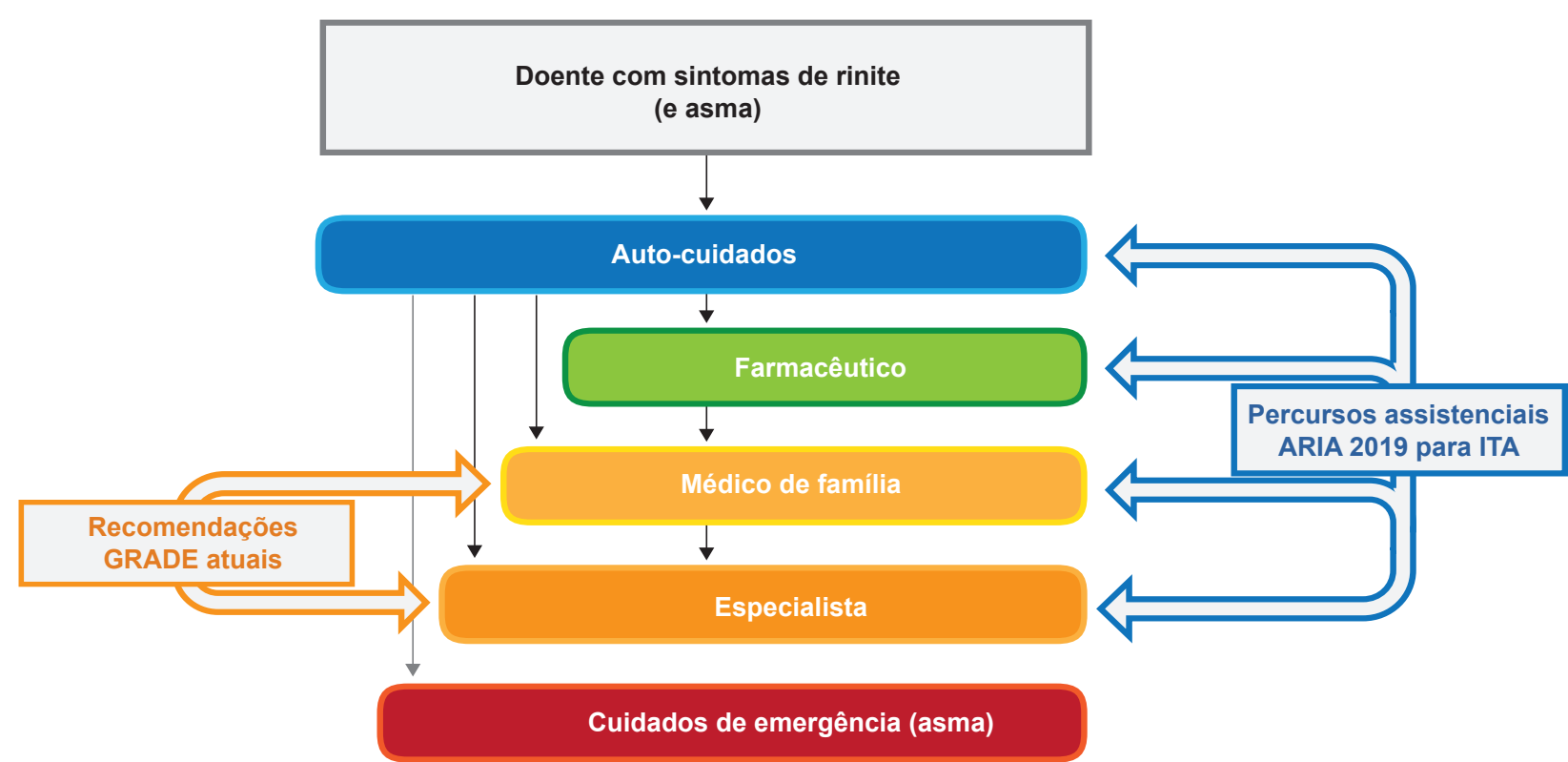

Figura 2 - Percursos assistenciais para a rinite alérgica e seu impacto na asma 2019

apresenta também uma significativa morbilidade, constituindo um peso financeiro importante nos custos de saúde que não pode ser subestimado. ${ }^{26-31}$

Recentemente, foi publicada uma revisão GA(2)LEN sobre o impacto económico do tratamento inadequado das doenças alérgicas na União Europeia. ${ }^{32}$ Esta iniciativa consistiu numa análise detalhada do custo do tratamento das doenças alérgicas nos trabalhadores do mercado europeu (incluindo a rinite alérgica) tendo-se estimado que os custos indiretos evitáveis por doenças alérgicas insuficientemente tratadas na união europeia variam entre 55 a 151 mil milhões de euros $(€)$ por ano, devido ao absentismo e ao presenteísmo. $\mathrm{O}$ tratamento adequado, usando tratamentos custo-efetivos, equivaleria a uma poupança média de $€ 142$ mil milhões por ano. Em Portugal, no entanto, não existem, actualmente, dados que estimem todos os custos associados à rinite alérgica. Esta patologia é abordada de modo indireto nas normas da Direção Geral de Saúde $N^{\circ}$ 61 de 2011 relativa à prescrição de exames laboratoriais e na norma $\mathrm{N}^{\circ} 6$ de 2018 relativa à asma.

Uma maior consciencialização sobre os efeitos prejudiciais da rinite alérgica na qualidade de vida dos doentes e o seu considerável custo devem incentivar o diagnóstico e o tratamento precoce, a fim de minimizar o fardo desta doença e garantir resultados mais custo-efetivos.

\subsection{Actividades ARIA}

A iniciativa Allergic Rhinitis and its Impact on Asthma (ARIA) , tem vindo a desenvolver e a promover a disseminação de orientações baseadas em evidência na área da rinite alérgica e da asma ao longo de mais de duas décadas, apoiando projetos piloto nessas áreas. Desde os primeiros passos do ARIA que especialistas Portugueses estão envolvidos e colaboram ativamente com esta iniciativa, participando na elaboração dos documentos de consenso ${ }^{33-35}$ e na sua implementação a nível local, nomeadamente com a tradução e distribuição do livro de bolso relativo às recomendações ARIA de 2001, promovidos pela Sociedade Portuguesa de Alergologia e Imunologia Clínica (SPAIC). ${ }^{36}$ Esta divulgação tem sido potenciada com a inclusão de recursos educacionais sobre a iniciativa ARIA em cursos de pós-graduação (p. ex.: nos cursos de pós-graduação de Doenças Respiratórias da Escola de Medicina da Universidade do Minho (três edições) e em cursos de doenças respiratórias para médicos de família (p. ex.: das escolas da Associação Portuguesa de Medicina Geral e Familiar, realizados anualmente desde 2011).

Adicionalmente, Portugal tem tido uma participação ativa em diversos projetos promovidos pelo ARIA que pretendem a implementação de cuidados de saúde apoiados por tecnologia móvel, com aquisição de evidência do mundo real [real world evidence (RWE)] e suporte à personalização e melhoria dos cuidados prestados aos doentes com RA. Dessas iniciativas salientam-se:

- Participação na tradução para Português da aplicação MASK-Air (anteriormente Diário de Alergia) disponível em Portugal desde 2016. Foram implementadas diversas atividades de disseminação e promoção da utilização da app, com divulgação nos meios de comunicação social, nas redes sociais e a nível dos cuidados de saúde. Esta divulgação permitiu que Portugal estivesse entre os países com maior número de utilizadores. ${ }^{37-39}$

- Participação nos estudos TWINNING que visam melhorar a compreensão, o diagnóstico e a abordagem terapêutica da rinite alérgica junto da população adulta e idosa. O estudo TWINNING MACVIA-ARIA utilizou a app Diário de Alergia, incluindo escalas visuais analógicas de sintomas e impacto da doença alérgica, em 23 locais de referência ou regiões na Europa, América do Sul e Oceania. ${ }^{1,40}$ Em Portugal, o TWINNING MACVIA-ARIA está a ser 
desenvolvido numa parceria nacional que envolve um conjunto alargado de instituições e unidades de saúde.

- Participação no desenho e implementação do estudo ARIA nas farmácias, a decorrer atualmente, que pretende verificar a implementação das orientações de 2019 da iniciativa ARIA, específicas para as farmácias comunitárias, no que diz respeito particularmente ao aconselhamento em automedicação para o doente com RA.

\section{Nova geração de recomendações ARIA-GRADE}

O tratamento farmacológico na RA tem como objetivo o controlo da patologia ${ }^{41}$ e a sua seleção depende: (i) da capacitação e das preferências do doente, (ii) dos sintomas predominantes, da sua gravidade e da multimorbilidade, (iii) da eficácia e segurança do tratamento, ${ }^{41}$ (iv) da rapidez de ação do tratamento, (v) do tratamento atual, (vi) da resposta aos tratamentos realizados anteriormente, (vii) do impacto no sono e na produtividade laboral, ${ }^{42,43}$ (viii) das estratégias de autogestão da doença e (ix) da utilização de recursos.

A metodologia GRADE (Grading of Recommendations Assessment, Development and Evaluation) considera, de modo explícito, a análise de evidência proveniente de todos os tipos de estudos. No entanto, as recomendações clínicas geralmente baseiam-se apenas nos resultados dos ensaios clínicos randomizados (RCT). A GRADE considera ainda relevante a evidência sobre valores e preferências, aceitabilidade e viabilidade ou comparabilidade dos resultados. Observa-se uma tendência crescente na utilização da RWE para informar a prática clínica, especialmente devido às limitações relacionadas com a aplicabilidade dos resultados dos $\mathrm{RCT}^{42}$ É desejável que o conhecimento produzido por RCT e por RWE seja utilizado em conjunto.

Durante a reunião de Paris, foram desenvolvidas as recomendações mais recentes para o tratamento farmacológico da RA, ${ }^{44}$ tendo como base as recomendações então vigentes baseadas na GRADE para RA, ${ }^{35,42,43,45}$ avaliadas através de RWE adquirida por tecnologia móvel, ${ }^{1,2,46,47}$ e utilizando a evidência resultante de estudos de câmara de exposição alergénica. Este conjunto alargado de conhecimento foi utilizado para refinar o algoritmo MASK para o tratamento da RA proposto anteriormente por um grupo de consenso. ${ }^{48}$

\subsection{Evidência considerada para o desenvolvimento do PAI ARIA}

Assim, para o desenvolvimento do PAI ARIA foram considerados i) o algoritmo MASK para tratamento farmacológico da RA; ii) as diretrizes ARIA 2010, a sua revisão de 2016 e os Practice Parameters de 2017 dos EUA; iii) estudos sobre o início de ação farmacológica em câmara de exposição a alergénios (CEA) e iv) RWE proveniente de tecnologia móvel.

\subsubsection{Algoritmo MASK para tratamento farmacológico da RA}

O algoritmo MASK, baseado na escala visual analógica (VAS), foi concebido ${ }^{48}$ e incluído numa ferramenta digital ${ }^{49}$ para seleção e ajuste (com base no controlo) do tratamento farmacológico para doentes com RA. (Fig.s 3A e 3B).

\subsubsection{ARIA 2010, revisão de 2016 e US Practice Para- meters 2017}

Diversas revisões ${ }^{41}$ e recomendações ${ }^{35,43,45,48}$ têm proposto comparações entre os medicamentos usados na RA, apesar de existirem poucos RCT que os comparem head-to-head. ${ }^{50-52}$ Um estudo de avaliação de tecnologias de saúde concluiu que a maioria dos medicamentos usados na RA têm efeitos semelhantes..$^{53}$ No entanto, este estudo utilizou um método demasiado restritivo, que não permitiu diferenciar os medicamentos entre si.

A revisão ARIA $2016^{35}$ e os Practice Parameters de 2017 dos EUA ${ }^{45}$ foram desenvolvidos independentemente, utilizando a mesma abordagem metodológica: GRADE. Ambos analisaram as mesmas perguntas e consideraram dois outcomes principais no tratamento da rinite moderada-grave: eficácia e rapidez de ação (Tabelas 1 e 2).

A revisão ARIA $2016^{35}$ e os US Practice Parameters $2017,{ }^{45}$ ambos baseados principalmente em RCTs, suportam o algoritmo MASK. ${ }^{45,48}$

\subsubsection{Início de ação dos medicamentos: estudos em CEA}

A Food and Drug Administration (FDA) propôs três tipos de estudos para avaliar o início de ação dos medicamentos para a RA ${ }^{54}$ : os RCTs de fase III em dupla ocultação, os estudos realizados em campo, e os estudos em CEA. Os estudos em CEA têm a vantagem de poder demonstrar em minutos o início de eficácia dos medicamentos. ${ }^{55}$

Foram testados vários medicamentos orais e intranasais nas CEA de Ontário ${ }^{56-59}$ e Viena. ${ }^{60-62}$ Os estudos na CEA de Ontário mostraram o início de ação rápido da azelastina e das suas combinações, incluindo a combinação fixa intranasal de cloridrato de azelastina e propionato de fluticasona (MP-AzeFlu). Os restantes anti-histamínicos $\mathrm{H} 1$ intranasais têm um início de ação mais lento. Os corticosteroides intranasais (INCS) (isolados ou combinados com anti-histamínicos $\mathrm{H} 1$ orais) só são eficazes cerca de duas horas após a administração. ${ }^{57}$ Os estudos na CEA de Viena mostraram que a azelastina e a levocabastina/furoato de fluticasona (FF) são os medicamentos com início de ação mais rápido, quando comparados com os anti-histamínicos $\mathrm{H} 1$ orais e ICNS.

\subsubsection{Evidência do mundo real usando tecnologia mó- vel}

As diretrizes 2019 do ARIA usaram as recomendações GRADE complementadas com RWE obtida por ferramentas de mHealth com o objetivo de confirmar e aprimorar tanto essas recomendações como o algoritmo MASK. Apesar de haver muitas ferramentas de mHealth disponíveis 


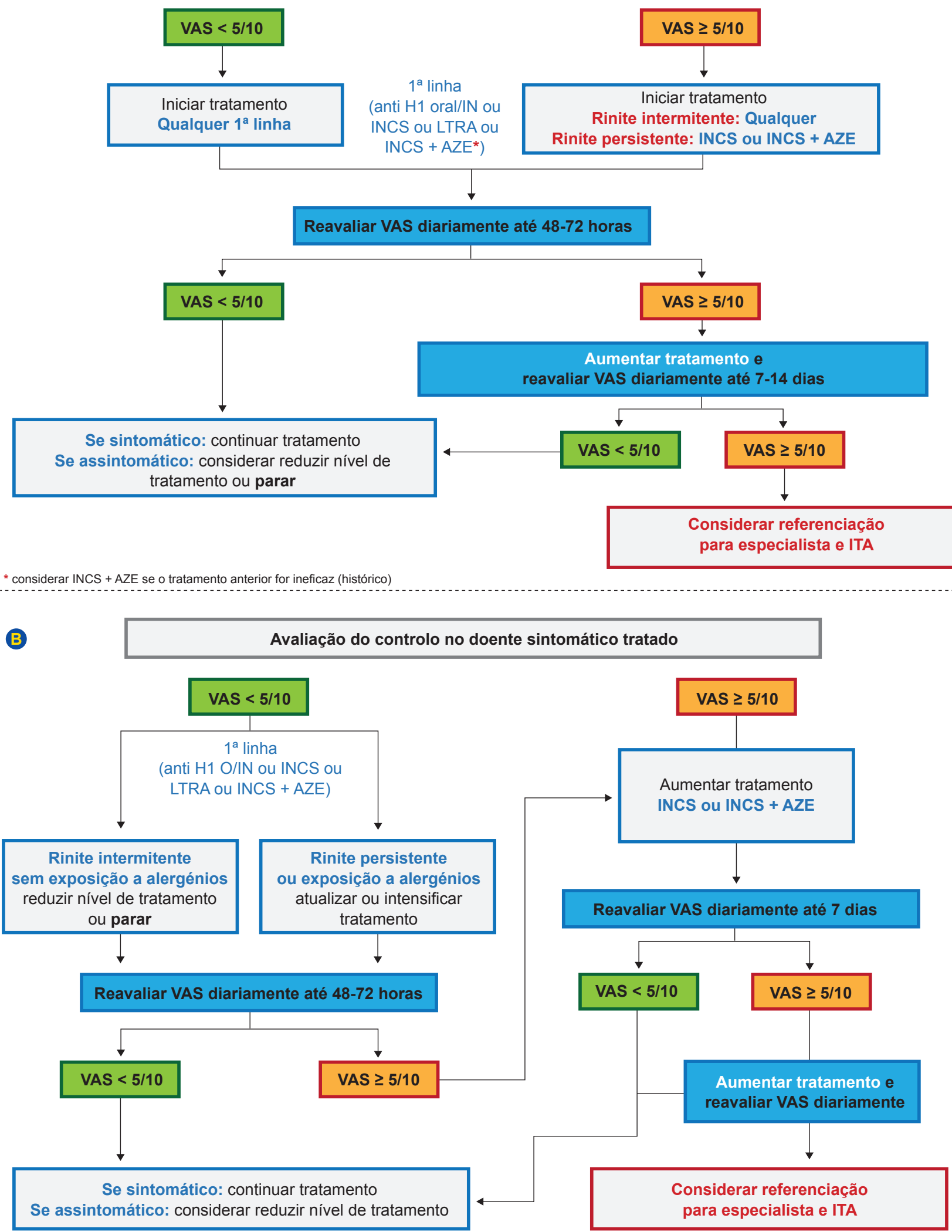

Figura 3 - (A) Algoritmo de tratamento para doentes sintomáticos não tratados, baseado na escala visual analógica (adolescentes e adultos); (B) Algoritmo de tratamento para doentes sintomáticos já tratados farmacologicamente, baseado na escala visual analógica (adolescentes e adultos) 
Tabela 1 - Recomendações gerais do ARIA $2016^{35}$

\begin{abstract}
Nos doentes com RA sazonal, foi recomendada uma combinação de INCS + (OAH) ou INCS em monoterapia, sendo que o potencial benefício global pode não justificar a utilização de recursos adicionais.

Em doentes com RA perene, utilizar apenas INCS em vez de uma combinação de INCS + OAH.

Nos doentes com RA sazonal, é sugerida uma combinação de INCS + INAH ou INCS em monoterapia, a selecionar de acordo com as preferências do doente. No início do tratamento (primeiras duas semanas), a combinação de INCS + INAH pode atuar mais rapidamente do que o INCS em monoterapia, podendo, portanto, ser preferida por alguns doentes. A terapia combinada pode ser uma escolha razoável em situações em que o seu custo adicional é reduzido. No entanto, em Portugal não está disponível a combinação INCS+INAH num mesmo dispositivo, não existindo informação suficiente para afirmar que a utilização de INCS e INAH em dispositivos diferentes terão resultados semelhantes à INCS+INAH.
\end{abstract}

Em doentes com RA perene, sugerimos a combinação de INCS + INAH ou INCS em monoterapia.

O nível de evidência foi baixo ${ }^{2,4}$ ou muito baixo ${ }^{1,6}$ para todas estas recomendações.

RA: rinite alérgica; INCS: corticosteróide intranasal; OAH: anti-histamínico oral; INAH: anti-histamínico intranasal

Tabela 2 - Recomendações clínicas relevantes dos Practice Parameters de 2017 dos EUA ${ }^{45}$

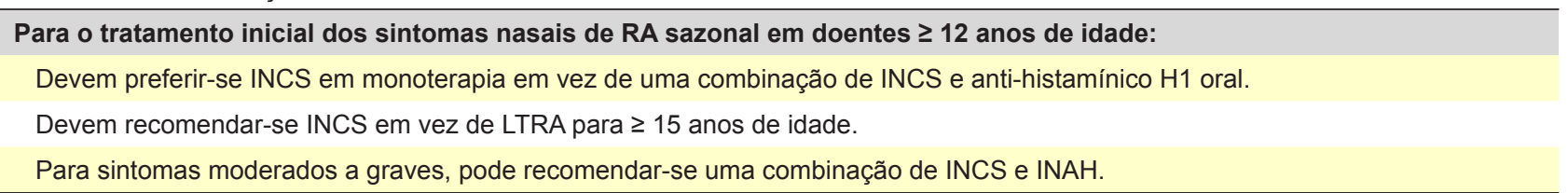

RA: rinite alérgica; INCS: corticosteróide intranasal; LTRA: antagonistas dos receptores dos leucotrienos; INAH: anti-histamínico intranasal

Tabela 3 - Resultados da RWE para o tratamento da RA

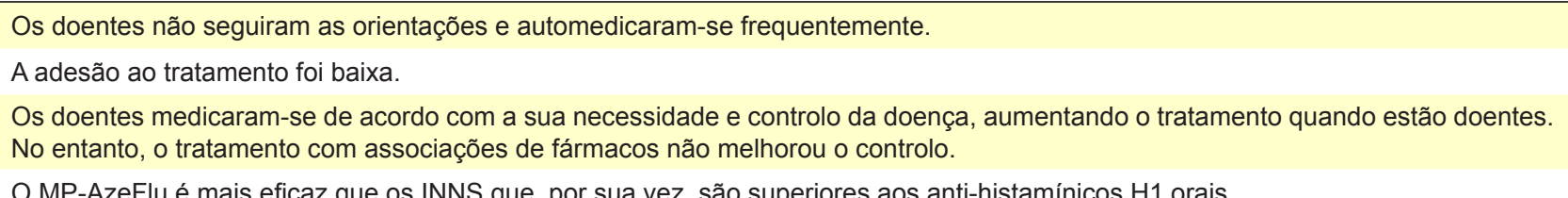

O MP-AzeFlu é mais eficaz que os INNS que, por sua vez, são superiores aos anti-histamínicos H1 orais.

RWE: evidência do mundo real; RA: rinite alérgica; INCS: corticosteróide intranasal

para a RA, ${ }^{63}$ apenas o MASK apresentou dados sobre medicamentos que constituem RWE. 1,62 Um resumo dos seus principais resultados está apresentado na Tabela 3.

Embora não seja possível a demonstração direta do grau de adesão ao tratamento - uma vez que os utilizadores do MASK não reportaram dados diariamente e podem não ter reportado todos os medicamentos utilizados - a adesão avaliada por meio da taxa de posse de medicamentos e da proporção de dias cobertos com medicação foi inferior a $5 \%{ }^{64}$

\section{Limitações da tecnologia móvel}

Tal como ocorre com todos os estudos que recorrem a auto-resposta voluntária dos participantes, podem ter ocorrido vieses de amostragem, erros de classificação dos resultados, e, devido a restrições éticas, existir pouca informação disponível sobre as características do doente ou das condições de resposta. Acresce ainda que os utilizadores de aplicações não são representativos da população de doentes com RA.

A análise dos dados do MASK considerou cada dia de cada doente de forma independente, isto é, como num estudo transversal. ${ }^{1}$ Foi usada esta abordagem devido à ausência de um padrão claro de tratamento e por não ser viável efetuar uma análise longitudinal devido ao uso intermitente da App pela maioria dos utilizadores.

Embora diagnóstico de RA não tenha sido confirmado pelo médico, é provável que a maioria dos utilizadores do MASK tivessem rinite (alérgica ou não alérgica). ${ }^{1}$

Apesar das limitações identificadas, a tecnologia móvel está a tornar-se uma ferramenta cada vez mais relevante para a compreensão e gestão da RA, acrescentando novos dados que, de outro modo, não eram passíveis de ser coIhidos. ${ }^{1,65,66}$

Outros estudos de RWE utilizando tecnologia móvel

Tanto quanto sabemos, não existem outros estudos de $m$ Health que tenham avaliado a eficácia de diferentes medicamentos para RA em grande escala.

\subsubsection{Desfasamento entre as perspetivas do doente e do médico}

Pensa-se que existe um elevado grau de desfasamento entre a prescrição médica e o comportamento do doente no tratamento da RA polínica tal como demonstrado noutras áreas médicas. ${ }^{67} \mathrm{E}$ frequente que a prescrição médica seja feita para a totalidade da época polínica, recomendando o uso de medicação mesmo em dias em que os sintomas são ligeiros. No entanto, a maioria dos doentes utiliza os medicamentos em função dos sintomas, apenas quando o controlo da RA piora, não seguindo, portanto, as recomendações. ${ }^{1,68}$ Os próprios médicos que têm RA, quando tratam a sua própria RA, comportam-se de modo idêntico aos restantes doentes. ${ }^{69}$ 
Tabela 4 - Recomendações para seleção de fármacos para rinite alérgica

\begin{tabular}{|c|c|c|c|}
\hline & GRADE & $\begin{array}{l}\text { RWE proveniente } \\
\text { de mHealth }\end{array}$ & CEA \\
\hline $\begin{array}{l}\text { Os anti-histamínicos } \mathrm{H} 1 \text { orais são menos potentes que os } \\
\text { INCS mas muitos doentes preferem tratamentos orais }\end{array}$ & $\begin{array}{c}\text { Nenhuma informação sobre a } \\
\text { preferência do doente }\end{array}$ & 38,70 & \\
\hline $\begin{array}{l}\text { Os anti-histamínicos H1 intranasais são menos eficazes do } \\
\text { que os INCS }\end{array}$ & 43 & 38,70 & \\
\hline $\begin{array}{l}\text { Os anti-histamínicos H1 intranasais são eficazes em poucos } \\
\text { minutos }\end{array}$ & 43 & 38 & 57,61 \\
\hline Os INCS são medicamentos potentes & 43,45 & 38,70 & \\
\hline $\begin{array}{l}\text { O início de ação dos INCS demora algumas horas a alguns } \\
\text { dias (exceto para a ciclesonida que é eficaz mais rapidamente) }\end{array}$ & 43 & & 60,71 \\
\hline $\begin{array}{l}\text { A combinação de INCS e anti-histamínicos H1 orais não } \\
\text { oferece vantagem sobre os INCS em monoterapia }\end{array}$ & 35,45 & 38,70 & \\
\hline $\begin{array}{l}\text { A combinação de INCS e anti-histamínicos H1 intranasais é } \\
\text { mais potente que os INCS em monoterapia }\end{array}$ & $\begin{array}{l}\text { SIM em doentes graves: }{ }^{45} \\
\text { Com algumas restrições } \\
\text { dependendo dos custos: }{ }^{35}\end{array}$ & 38,70 & \\
\hline $\begin{array}{l}\text { A combinação de INCS e anti-histamínicos H1 intranasais é } \\
\text { eficaz em poucos minutos }\end{array}$ & & 38 & $58,60,63$ \\
\hline $\begin{array}{l}\text { Os antagonistas dos leucotrienos são menos potentes que os } \\
\text { INCS }\end{array}$ & 35,45 & 38 & \\
\hline
\end{tabular}

RWE: evidência do mundo real; CEA: câmara de exposição a alergénios; INCS: corticosteróide intranasal

Tabela 5 - Recomendações para a farmacoterapia na rinite alérgica

Os anti-histamínicos $\mathrm{H} 1$ orais ou intranasais são menos eficazes do que os INCS no controlo de todos os sintomas da rinite. ${ }^{43,72-74}$ No entanto, são eficazes em muitos doentes com rinite leve/moderada e muitos doentes preferem medicamentos orais a intranasais com maior rapidez de ação.

As comparações entre os anti-histamínicos $\mathrm{H} 1$ orais e intranasais diferem entre as várias recomendações, não tendo sido possível chegar a conclusões definitivas.

Os INCS são o tratamento de primeira linha em doentes com rinite grave, necessitando, contudo, de alguns dias para atingir a sua eficácia máxima.

A combinação de um anti-histamínico H1 oral com um INCS não tem eficácia superior aos INCS em monoterapia, ${ }^{35,45}$ apesar de ser prática comum.

O MP-AzeFlu - o propionato de fluticasona intranasal combinado com a Azelastina em dispositivo único - é mais eficaz do que a monoterapia com INCS, estando indicado quando a monoterapia com INCS é considerada inadequada, ${ }^{35,45,51,75-78}$ para doentes com RA grave, ou para doentes que necessitam de um alívio rápido dos sintomas. ${ }^{35,45} \mathrm{Um}$ estudo em câmara de exposição a alergénios confirmou o início de ação rápido dessa combinação. ${ }^{58,79}$ Atualmente esta combinação terapêutica não está disponível em Portugal.

Todos os medicamentos recomendados são considerados seguros na dose habitual. Os anti-histamínicos $\mathrm{H} 1$ orais de primeira geração são sedativos e devem ser evitados. ${ }^{80} \mathrm{O}$ uso prolongado de vasoconstritores nasais deve ser também evitado.

Os corticosteróides intramusculares depot são contraindicados para o tratamento da RA.

INCS: corticosteróide intranasal; RA: rinite alérgica

\subsection{Recomendações ARIA-GRADE 2019}

O algoritmo proposto apresenta uma abordagem passo-a-passo para a seleção farmacológica na RA tendo como base as recomendações GRADE ajustadas à evidência proveniente de RWE e aos resultados dos estudos em CEA (Tabela 4). ${ }^{44}$

A abordagem proposta i) confirma a validade da maioria das recomendações ARIA para a RA, baseadas na metodologia GRADE, ii) usa RWE, permite corroborar alguma da evidência anterior que tinha menor robustez e iii) fornece novas perspetivas sobre os tratamentos, em particular:

- A eficácia da combinação de anti-histamínicos H1 orais e INCS não foi superior à dos INCS em monoterapia.

- A eficácia da combinação de anti-histamínicos H1 intranasais e INCS foi superior à dos INCS em monoterapia.
- Os medicamentos que contêm anti-histamínicos H1 intranasais são eficazes em poucos minutos.

- Os custos mais elevados de uma combinação fixa de INCS e anti-histamínicos H1 intranasais justifica-se quando os sintomas não são controlados de outra forma ${ }^{35}$

Em geral, as recomendações para a RA baseadas na metodologia ARIA-GRADE concordam nos pontos detalhados na Tabela $5.35,43,45,48$

\section{Percursos de tratamento ARIA 2019 para a imunote- rapia com alergénios (ITA)}

A imunoterapia com alergénios (ITA) é uma opção terapêutica comprovada para o tratamento da RA e/ou asma, podendo ser feita por via sublingual (SLIT) ou subcutânea (SCIT). ${ }^{43,80-86}$ A eficácia demonstrada em RCT em dupla ocultação e controlados por placebo foi confirmada em 
estudos utilizando bases de dados de prescrições e comprovada na vida real. ${ }^{87} \mathrm{Na}$ maioria dos países, a ITA tem um custo mais elevado do que outros tratamentos médicos para a RA ou asma ${ }^{87-89}$ e, portanto, deve ser considerada no contexto de uma abordagem terapêutica estratificada. ${ }^{33}$ As recomendações existentes sobre ITA ${ }^{43,80-85}$ são complexas, não propõem PAls e utilizam métodos muito variados para obtenção e avaliação da evidência. O ARIA 2019 produziu PAls para SCIT e SLIT ${ }^{90}$ que estão resumidos neste artigo.

\subsection{Alergénios a utilizar \\ Extrato relevante}

A decisão de prescrever ITA deve ser baseada na relevância do alergénio, tendo em conta os sintomas durante a exposição alergénica, a demonstração de sensibilização e disponibilidade de extratos de boa qualidade, sempre que possível padronizados..$^{81,91}$

\section{Extrapolação para produtos não testados}

Os produtos de ITA têm de demonstrar eficácia e segurança em conformidade com os requisitos regulamentares. ${ }^{92-94}$

\section{Mistura de extratos}

Não há evidência de que as misturas de alergénios tenham o mesmo efeito que a administração individual de cada alergénio. A mistura pode causar a diluição e degradação dos alergénios devido à atividade enzimática que alguns apresentam. A Agência Europeia de Medicamentos (EMA) recomenda apenas a mistura de alergénios de grupos homólogos. ${ }^{94}$

\subsection{Segurança}

\subsubsection{Imunoterapia subcutânea (SCIT)}

Uma reação típica (reação local) manifesta-se por rubor e edema no local da injeção imediatamente ou várias horas após a administração. Por vezes, podem ocorrer reações sistémicas que se manifestam, por exemplo, com rinite, agravamento de asma ou urticária. ${ }^{95}$ Apesar de reações graves à SCIT serem muito raras, requerem tratamento imediato. As reações mais graves desenvolvem-se nos primeiros 30 minutos após a injeção, pelo que se recomenda que os doentes permaneçam sob vigilância médica durante pelo menos 30 minutos após a administração.

\subsubsection{Imunoterapia sublingual (SLIT)}

As gotas ou comprimidos de alergénios têm um perfil de segurança mais favorável do que a SCIT. Após a primeira dose, que deve ser feita sob supervisão médica, a SLIT pode ser administrada em casa. A maioria dos eventos adversos é local (prurido na boca, edema dos lábios, náuseas), não implicando alteração do esquema terapêutico, e resolvendo habitualmente de forma espontânea em poucos dias. A gravidade dos efeitos secundários locais é classificada de acordo com a persistência e o impacto na qualidade de vida. ${ }^{95,96}$

\subsection{Perspetiva do doente}

A perspetiva do doente deve ser sempre considerada, de forma a permitir uma abordagem personalizada integrada numa decisão terapêutica partilhada (DTP). Existem estudos de vida real com resultados contraditórios no que respeita à avaliação do nível de conhecimento, perceções, expectativas e satisfação relacionadas com a ITA. ${ }^{97,98}$ No entanto, a maioria dos estudos reporta falta de informação dos doentes alérgicos, devendo ser envidados esforços no sentido de melhorar a comunicação, de forma a promover o aumento do conhecimento e da satisfação dos doentes. ${ }^{99,100}$

A adesão à ITA é crucial para a sua eficácia. No entanto, a não adesão e a descontinuação prematura são comuns. ${ }^{101}$ Há resultados controversos sobre a taxa de adesão a ITA, mas pensa-se que seja baixa. ${ }^{102} \mathrm{Um}$ artigo recente sugere que um horário reservado para as administrações de ITA, reduz o tempo de espera, tornando a vigilância mais efetiva e aumentando o foco no doente a fazer ITA; este acompanhamento mais próximo poderá ajudar a aumentar a adesão ao tratamento. ${ }^{101}$

\subsection{Perspetiva do farmacêutico}

A maioria dos doentes gere a sua RA sem ter contato suficiente com o seu médico. ${ }^{103}$ Os farmacêuticos são os profissionais de saúde mais acessíveis ao público e a RAé uma das doenças mais frequentemente geridas por farmacêuticos. ${ }^{104,105} \mathrm{Em}$ muitos países, os produtos de ITA estão disponíveis em farmácias comunitárias e os farmacêuticos devem estar bem informados sobre este tratamento. Nestes países, os farmacêuticos podem desempenhar um papel importante na educação dos doentes sobre a adesão, os compromissos envolvidos na ITA, bem como os seus riscos e benefícios. Em Portugal, as normas de prescrição e as normas de dispensa da Autoridade Nacional do Medicamento e Produtos de Saúde, I.P. (INFARMED) prevêem que a dispensa seja feita nas farmácias comunitárias, embora atualmente e esteja apenas disponível em farmácia comunitária um comprimido sublingual para ITA a pólenes de gramíneas. ${ }^{106,107}$ É, pois, de esperar que o circuito de disponibilização das outras formas de ITA disponíveis em Portugal passe também a ser feito através da farmácia comunitária, o que permitirá um envolvimento mais efetivo dos farmacêuticos na educação destes doentes.

\subsection{Perspetiva do médico de família}

Em muitos países, o diagnóstico e o tratamento das patologias alérgicas ocorrem fundamentalmente nos cuidados de saúde primários. ${ }^{108,109}$ A continuidade dos cuidados, a acessibilidade e o caráter holístico dos cuidados de saúde primários é vital na gestão da RA e na decisão terapêutica partilhada centrada no doente. ${ }^{110,111}$ No entanto, poucos médicos de Medicina Geral e Familiar (MGFs) recebem formação formal pré ou pós-graduada em Alergologia. ${ }^{112}$ A SCIT também pode ser administrada nos cuidados de saúde primários sendo os riscos associados minimizados quando feita por profissionais treinados, num ambiente adequado 
e com instalações e equipamento apropriado para o tratamento de reações sistémicas. ${ }^{113,114}$

\subsection{Abordagem prática para estratificação de doentes na ITA}

A ITA deve ser prescrita por um médico com formação específica em Imunoalergologia. A decisão terapêutica partilhada é essencial para iniciar ITA. Em muitos países, a ITA é um tratamento com custos importantes, devendo ser proposto a doentes selecionados. O custo da ITA pode variar entre cerca de $€ 300$ até mais de $€ 1000$ por ano, ao qual acresce o custo da administração em unidade de saúde sob vigilância médica, no caso da SCIT. Apesar da ITA ser custo-efetiva, ${ }^{89,115,116}$ em Portugal deixou de ser comparticipada em 2014. Os doentes devem saber se a ITA está coberta pelo seu subsistema ou seguro de saúde e quais os custos que serão pagos diretamente por si (não cobertos pelo financiador).

\subsubsection{Estratificação de doentes alérgicos para ITA}

O papel da medicina de precisão na seleção de um regime de ITA apropriado para um doente específico foi proposto por um grupo de especialistas (Tabela 6). ${ }^{117,118}$

Foi proposto um fluxograma de medicina de precisão para a ITA (Fig. 4). ${ }^{87,117}$ Antes de iniciar ITA é necessário considerar o grau de controlo atingido com a terapêutica farmacológica. Em alguns casos, a ITA pode ser proposta a doentes cuja RA está controlada com a terapêutica farmacológica instituída mas têm crises de asma em determinadas condições ambientais específicas, por exemplo, em relação com exposição alergénica mais intensa. ${ }^{120,121}$ A ITA também deve ser considerada, mesmo na RA moderada, particularmente (mas não apenas) em doentes que tiveram agudizações de asma durante a época polínica e que vivem em regiões geográficas de maior risco para exposição aos pólenes a que são alérgicos.

\subsubsection{Multimorbilidade}

A multimorbilidade - coexistência de mais de uma doença alérgica no mesmo doente - é muito comum nas doenças alérgicas. Cerca de $85 \%$ dos doentes asmáticos sofrem também de RA. Por outro lado, $20 \%$ - 30\% dos doentes com RA têm asma. A coexistência de RA em doentes com asma aumenta a gravidade da asma. ${ }^{122}$ A ITA pode controlar a multimorbilidade da RA, conjuntivite e asma. A multimorbilidade foi reconhecida como uma indicação para a SLIT a ácaros nas condições de autorização de comercialização de ITA em comprimidos sublinguais. ${ }^{123}$

\subsubsection{Crianças}

A ITA é eficaz em crianças, ${ }^{124}$ podendo o seu efeito persistir durante longos períodos após o término do tratamento. ${ }^{125}$ Estudos recentes de SLIT, ${ }^{126}$ um estudo anterior de SCIT a pólen de gramíneas, ${ }^{127}$ e uma meta-análise, ${ }^{128}$ forneceram alguma evidência de que a ITA pode atrasar ou prevenir o aparecimento de asma em crianças com rinite. No entanto, a meta-análise mostrou que a redução do risco de desenvolver asma a curto prazo é limitada e com benefícios pouco claros a longo prazo. ${ }^{128}$ Assim, a ITA pode ser iniciada em crianças com RA moderada/grave que não é controlada pela farmacoterapia. Nas crianças sem asma, a possibilidade de prevenir o início da asma deve ser tida em consideração, embora sejam necessários mais estudos para que se possa dar uma indicação sem reservas.

\subsubsection{Imunoterapia com alergénios em geriatria}

As características imunológicas e alergológicas dos doentes alérgicos geriátricos diferem das dos adultos jovens e dos de meia-idade. Estudos com evidência limitada sugerem que a ITA pode ser eficaz nesta população ${ }^{129}$; no entanto, é necessária mais informação.

\section{7. mHealth na abordagem da medicina de precisão ITA}

\section{Estratificação dos doentes}

A estratificação dos doentes pode ser facilitada através da utilização de diários eletrónicos disponibilizados no telemóvel ${ }^{47,130}$ ou noutros instrumentos de $\mathrm{mHealth}$. Após um período de preenchimento, os médicos podem avaliar (i) a gravidade e controlo da doença, (ii) a associação dos sintomas a épocas polínicas ou à exposição a outros alergénios, (iii) a adesão do doente ao tratamento farmacológico, (iv) a duração dos sintomas não controlados e (v) o impacto na produtividade no trabalho ou na escola. Um sistema eletrónico de apoio à decisão clínica poderá, no futuro, auxiliar a estratificação de doentes para ITA. ${ }^{49,131}$

Tabela 6 - Medicina de precisão na indicação de ITA com aeroalergénios (adaptado ${ }^{88,118}$ )

\footnotetext{
1. Diagnóstico preciso com história, testes cutâneos por picada e/ou lgE específica e, se necessário, diagnóstico in vitro por componentes moleculares ${ }^{88,120}$. Em alguns casos podem ser necessários testes de provocação (nasal, conjuntival ou brônquico).

2. Indicações comprovadas: rinite alérgica, conjuntivite e/ou asma.

3. Sintomas de alergia induzidos predominantemente pela exposição a alergénios relevantes.

4. Estratificação de doentes: Mau controlo dos sintomas apesar de tratamento farmacológico adequado, prescrito de acordo com as diretrizes atuais e com adesão durante a época sintomática. Alteração da história natural da alergia.

5. A tecnologia móvel pode ser relevante para a estratificação dos doentes (biomarcador $m$ Health).

6. Demonstração da eficácia e segurança do produto com ensaios clínicos relevantes.

7. Decisão terapêutica partilhada: as perspetivas do doente (e do cuidador) são um componente essencial na decisão de iniciar ITA.

ITA: imunoterapia com alergénios
} 


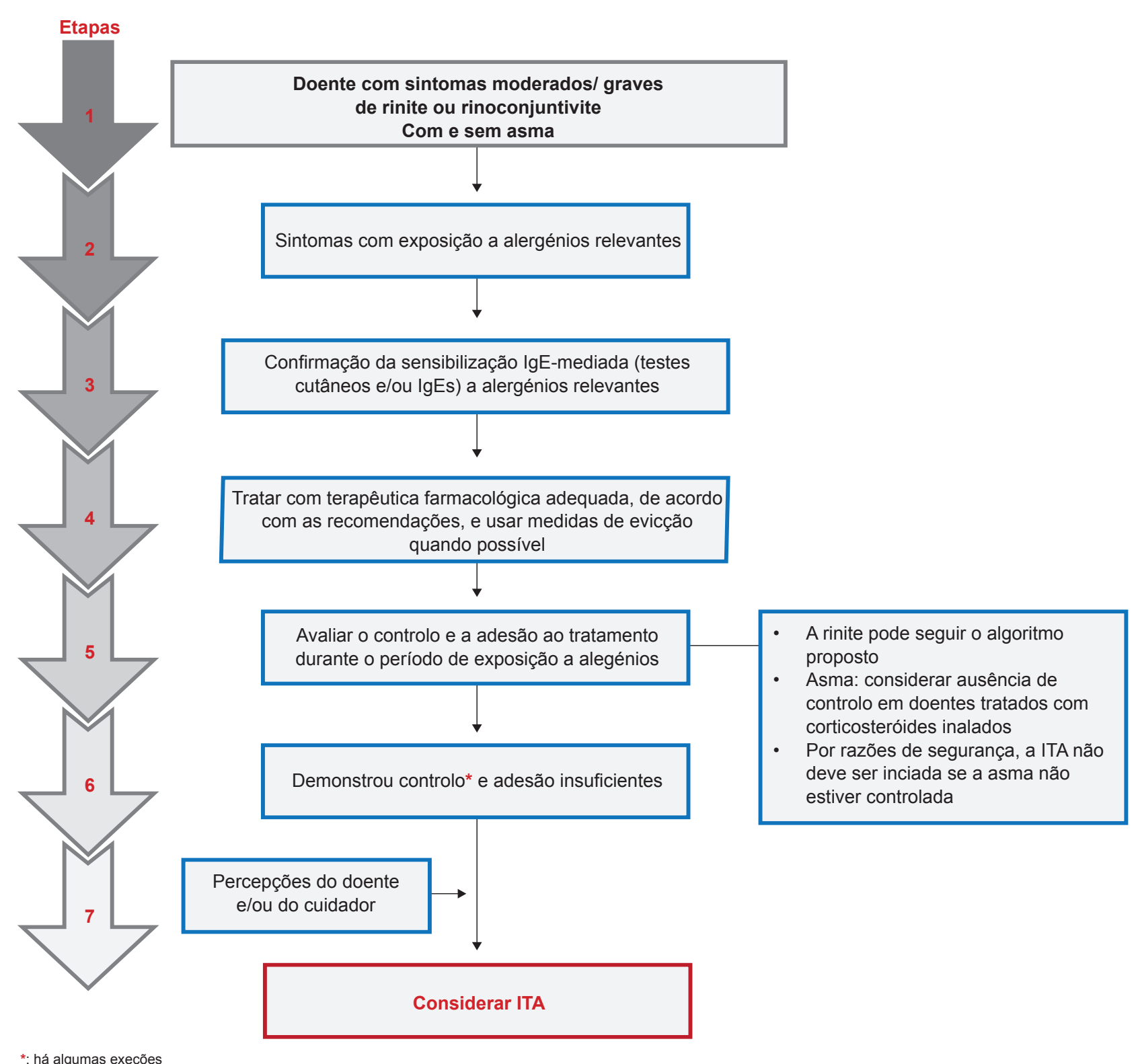

*. há algumas exeções

Figura 4 - Fluxograma de medicina de precisão para imunoterapia com alergénios (adaptado88,118)

\section{Acompanhamento de doentes sob ITA}

A mesma abordagem de $m$ Health pode ser proposta para o acompanhamento de doentes sob tratamento com ITA para avaliar sua eficácia. ${ }^{131}$

\section{CONCLUSÃO}

Esta nova geração de recomendações ARIA-GRADE constitui a primeira diretriz clínica que, além de usar a metodologia GRADE, integra evidência de RCTs, de RWE adquirida através de tecnologia $\mathrm{mHealth}$, e de estudos em CEA, podendo ser um modelo para outras doenças crónicas.

A terapêutica farmacológica na rinite alérgica tem como objetivo o controlo da doença, devendo ser adaptada às caraterísticas do doente, da doença e da resposta aos tratamentos prévios. Os algoritmos propostos, usando o resulta- do da escala visual analógica, apresentam uma abordagem passo a passo baseada na evidência para a seleção da terapêutica farmacológica na rinite alérgica. Adicionalmente, considera-se a ITA um exemplo de medicina de precisão, sendo um tratamento eficaz para as doenças alérgicas causadas por aeroalergénios. No entanto, o seu uso deve ser restrito a doentes cuidadosamente selecionados após estratificação, para os quais esteja disponível ITA eficaz e custo-efetiva.

Estas recomendações foram consideradas como 'boas práticas' dos cuidados integrados centrados no doente apoiados por sistemas digitais da Direção Geral de Saúde e de Segurança Alimentar da União Europeia (DG Santé) e representam a estratégia de gestão da mudança da fase 4 do ARIA. ${ }^{37}$ 


\section{PROTEÇÃO DE PESSOAS E ANIMAIS}

Os autores declaram que os procedimentos seguidos estavam de acordo com os regulamentos estabelecidos pelos responsáveis da Comissão de Investigação Clínica e Ética e de acordo com a Declaração de Helsínquia da Associação Médica Mundial actualizada em 2013.

\section{CONFIDENCIALIDADE DOS DADOS}

Os autores declaram ter seguido os protocolos do seu centro de trabalho acerca da publicação de dados.

\section{CONFLITOS DE INTERESSE}

João A. Fonseca: Recebeu fundos para apoio à investigação da Mundipharma e honorários por consultoria à AstraZeneca, Novartis e Mundipharma, fora do âmbito do presente trabalho e enquanto sócio de pequena-média empresa que desenvolve tecnologia móvel para a monitorização de doenças das vias respiratórias.

Tiago Taveira-Gomes: Recebeu honorários da AstraZeneca, BIAL, Daichii-Sankyo e MSD, a título privado e como orador, todos fora do âmbito do presente trabalho.

Ana Margarida Pereira: Não tem conflitos de interesses relacionados com o presente trabalho.

Manuel Branco-Ferreira: É o Presidente da Sociedade Portuguesa de Alergologia e Imunologia Clínica.

Pedro Carreiro-Martins: Não tem conflitos de interesses relacionados com o presente trabalho.

Magna Alves-Correia: Não tem conflitos de interesses relacionados com o presente trabalho.

Jaime Correia de Sousa: Recebeu honorários da Boehringer Ingelheim, GlaxoSmithKline, AstraZeneca e Mundipharma, a título privado e como orador, todos fora do âmbito do presente trabalho.

Elísio Costa: Não tem conflitos de interesses relacionados com o presente trabalho.

Olga Lourenço: Não tem conflitos de interesses relacionados com o presente trabalho.

Mário Morais-Almeida: Não tem conflitos de interesses relacionados com o presente trabalho.

Ana Morête: Não tem conflitos de interesses relacionados com o presente trabalho.

\section{REFERÊNCIAS}

1. Bousquet J, Arnavielhe S, Bedbrook A, Bewick M, Laune D, MathieuDupas E, et al. MASK 2017: ARIA digitally-enabled, integrated, personcentred care for rhinitis and asthma multimorbidity using real-worldevidence. Clin Transl Allergy. 2018;8:45.

2. Bousquet J, Anto JM, Annesi-Maesano I, Dedeu T, Dupas E, Pépin $\mathrm{JL}$, et al. POLLAR: Impact of air POLLution on Asthma and Rhinitis; a European Institute of Innovation and Technology Health (EIT Health) project. Clin Transl Allergy. 2018;8:36.

3. Bousquet JJ, Schünemann HJ, Togias A, Erhola M, Hellings PW, Zuberbier T, et al. Next-generation ARIA care pathways for rhinitis and asthma: a model for multimorbid chronic diseases. Clin Transl Allergy. 2019;9:44.

4. Campbell H, Hotchkiss R, Bradshaw N, Porteous M. Integrated care pathways. BMJ. 1998;316:133-7

5. Palmer K, Marengoni A, Forjaz MJ, Jureviciene E, Laatikainen T, Mammarella $F$, et al. Multimorbidity care model: recommendations from the consensus meeting of the Joint Action on Chronic Diseases and Promoting Healthy Ageing across the Life Cycle (JA-CHRODIS). Health
Frederico S Regateiro: Recebeu honorários da AstraZeneca, Novartis, Sanofi, GSK, Teva e Lusomedicamenta, a título privado e como orador, todos fora do âmbito do presente trabalho.

Ana Todo Bom: Não tem conflitos de interesses relacionados com o presente trabalho.

Claus Bachert: Recebeu honorários da Mylan, Sanofi, AstraZeneca, Novartis e GSK, a título privado e fora do âmbito do presente trabalho.

Oliver Pfaar: Recebeu bolsas e honorários a título privado da ALK-Abelló, Allergopharma, Stallergenes Greer, HAL Allergy Holding B.V./HAL Allergie $\mathrm{GmbH}$, Bencard Allergie GmbH/Allergy Therapeutics, Lofarma, ASIT Biotech Tools S.A., Laboratorios LETI/LETI Pharma e Anergis S.A., bolsas da Biomay, Circassia, GlaxoSmithKline, Pohl-Boskamp e Inmunotek S.L., honorários a título privado da MEDA Pharma/MYLAN, Mobile Chamber Experts (a GA2LEN Partner), Indoor Biotechnologies, Astellas Pharma Global, EUFOREA, NOVARTIS, SANOFI AVENTIS, Med Update Europe $\mathrm{GmbH}$ e streamedup! $\mathrm{GmbH}$, todos fora do âmbito do presente trabalho.

Dana Wallace: É ex-co-chair da Joint Task Force on Practice Parameters, um grupo de trabalho composto por12 membros da American Academy of Allergy, Asthma, and Immunology e do American College of Allergy, Asthma, and Immunology. Também recebeu apoio da ALK, fora do âmbito do presente trabalho.

Anna Bedbrook:- Não tem conflitos de interesses relacionados com o presente trabalho.

Wienczyslawa Czarlewski: Não tem conflitos de interesses relacionados com o presente trabalho.

Jean Bousquet: Recebeu honorários como orador da Chiesi, Cipla, Hikma, Menarini, Mundipharma, Mylan, Novartis, Purina,Sanofi-Aventis, Takeda, Teva e Uriach. Detém acções da KYomed INNOV.

\section{FONTES DE FINANCIAMENTO}

Este trabalho não recebeu qualquer tipo de suporte financeiro de nenhuma entidade no domínio público ou privado.

Policy. 2018;122:4-11.

6. Hujala A, Taskinen H, Rissanen S. How to support integration to promote care for people with multimorbidity in Europe? In: Richardson E, van Ginneken E, editors. European Observatory Policy Brief Series. Copenhagen: European Observatory on Health Systems and Policies; 2017.

7. European Innovation Partnership on Active and Healthy Ageing, Action Plan B3, Mechanisms of the Development of Allergy, WP 10, Global Alliance against Chronic Respiratory Diseases, Bousquet J, et al. Integrated care pathways for airway diseases (AIRWAYS-ICPs). Eur Respir J. 2014;44:304-23.

8. Hellings PW, Borrelli D, Pietikainen S, Agache I, Akdis C, Bachert C, et al. European summit on the prevention and self-management of chronic respiratory diseases: report of the European Union Parliament Summit (29 March 2017). Clin Transl Allergy. 2017;7:49.

9. Bousquet J, Onorato GL, Bachert C, Barbolini M, Bedbrook A, Bjermer $\mathrm{L}$, et al. CHRODIS criteria applied to the MASK (MACVIA-ARIA Sentinel NetworK) good practice in allergic rhinitis: a SUNFRAIL report. Clin 
Transl Allergy. 2017;7:37

10. Almeida MM, Loureiro C, Todo-Bom A, Nunes C, Pereira C, Delgado $\mathrm{L}$, et al. Rhinitis prevalence and characterization survey in primary care centres of mainland Portugal-ARPA study. Rev Port Imunoalergol. 2005;13:69-80.

11. Morais-Almeida M, Pite $\mathrm{H}$, Pereira AM, Todo-Bom A, Nunes C, Bousquet $\mathrm{J}$, et al. Prevalence and classification of rhinitis in the elderly: a nationwide survey in Portugal. Allergy. 2013;68:1150-7.

12. Morais-Almeida $M$, Nunes $C$, Gaspar A, Falcão $H$, Branco Ferreira $M$, Todo-Bom $A$, et al. Rinite em idade pré-escolar: prevalência e caracterização. Estudo ARPA Kids. Rev Port Imunoalergol. 2007;15:387410.

13. Todo-Bom A, Loureiro C, Almeida MM, Nunes C, Delgado L, CastelBranco G, et al. Epidemiology of rhinitis in Portugal: evaluation of the intermittent and the persistent types. Allergy. 2007;62:1038-43.

14. Bauchau V, Durham SR. Prevalence and rate of diagnosis of allergic rhinitis in Europe. Eur Respir J. 2004;24:758-64.

15. Morais-Almeida M, Santos N, Pereira AM, Branco-Ferreira M, Nunes C, Bousquet $\mathrm{J}$, et al. Prevalence and classification of rhinitis in preschool children in Portugal: a nationwide study. Allergy. 2013;68:1278-88.

16. Demoly $P$, Bossé I, Maigret P. Perception and control of allergic rhinitis in primary care. NPJ Prim Care Respir Med. 2020;30:37.

17. Gani F, Lombardi C, Barrocu L, Landi M, Ridolo E, Bugiani M, et al. The control of allergic rhinitis in real life: a multicenter cross-sectional Italian study. Clin Mol Allergy. 2018;16:4.

18. Nolte H, Nepper-Christensen S, Backer V. Unawareness and undertreatment of asthma and allergic rhinitis in a general population. Respir Med. 2006;100:354-62.

19. Bousquet PJ, Demoly P, Devillier P, Mesbah K, Bousquet J. Impact of allergic rhinitis symptoms on quality of life in primary care. Int Arch Allergy Immunol. 2013;160:393-400.

20. Bousquet J, Bullinger M, Fayol C, Marquis $P$, Valentin B, Burtin B Assessment of quality of life in patients with perennial allergic rhinitis with the French version of the SF-36 Health Status Questionnaire. J Allergy Clin Immunol. 1994;94:182-8.

21. Meltzer EO, Nathan RA, Seiner JC, Storms W. Quality of life and rhinitic symptoms: results of a nationwide survey with the SF-36 and RQLQ questionnaires. J Allergy Clin Immunol. 1997;99:S815-9.

22. Meltzer EO. Quality of life in adults and children with allergic rhinitis. J Allergy Clin Immunol. 2001;108:S45-53.

23. Roger A, Arcalá Campillo E, Torres MC, Millan C, Jáuregui I, Mohedano $\mathrm{E}$, et al. Reduced work/academic performance and quality of life in patients with allergic rhinitis and impact of allergen immunotherapy. Allergy Asthma Clin Immunol. 2016;12:40.

24. Vandenplas O, Suarthana E, Rifflart C, Lemière C, Le Moual N, Bousquet $\mathrm{J}$. The impact of work-related rhinitis on quality of life and work productivity: a general workforce-based survey. J Allergy Clin Immunol Pract. 2020;8:1583-91.e5.

25. Bédard A, Antó JM, Fonseca JA, Arnavielhe S, Bachert C, Bedbrook A, et al. Correlation between work impairment, scores of rhinitis severity and asthma using the MASK-air® App. Allergy. 2020;75:1672-88.

26. Blaiss MS. Allergic rhinitis: direct and indirect costs. Allergy Asthma Proc. 2010;31:375-80.

27. Malone DC, Lawson KA, Smith DH, Arrighi HM, Battista C. A cost of illness study of allergic rhinitis in the United States. J Allergy Clin Immunol. 1997;99:22-7.

28. Meltzer EO, Bukstein DA. The economic impact of allergic rhinitis and current guidelines for treatment. Ann Allergy Asthma Immunol. 2011;106:S12-6.

29. Cardell LO, Olsson P, Andersson M, Welin KO, Svensson J, Tennvall GR, et al. TOTALL: high cost of allergic rhinitis-a national Swedish population-based questionnaire study. NPJ Prim Care Respir Med. 2016;26:15082.

30. Avdeeva KS, Reitsma S, Fokkens WJ. Direct and indirect costs of allergic and non-allergic rhinitis in the Netherlands. Allergy. 2020;75:2993-6.

31. Colás C, Brosa M, Antón E, Montoro J, Navarro A, Dordal MT, et al. Estimate of the total costs of allergic rhinitis in specialized care based on real-world data: the FERIN Study. Allergy. 2017;72:959-66.

32. Zuberbier T, Lötvall J, Simoens S, Subramanian SV, Church MK. Economic burden of inadequate management of allergic diseases in the European Union: a GA(2) LEN review. Allergy. 2014;69:1275-9.

33. Bousquet J, Khaltaev N, Cruz AA, Denburg J, Fokkens WJ, Togias A, et al. Allergic Rhinitis and its Impact on Asthma (ARIA) 2008 update (in collaboration with the World Health Organization, GA(2)LEN and AllerGen). Allergy. 2008;63:S8-160.

34. Bousquet J, Schünemann HJ, Samolinski B, Demoly P, Baena-Cagnani
CE, Bachert C, et al. Allergic Rhinitis and its Impact on Asthma (ARIA): achievements in 10 years and future needs. J Allergy Clin Immunol. 2012;130:1049-62.

35. Brożek JL, Bousquet J, Agache I, Agarwal A, Bachert C, BosnicAnticevich S, et al. Allergic Rhinitis and its Impact on Asthma (ARIA) guidelines-2016 revision. J Allergy Clin Immunol. 2017;140:950-8.

36. Sociedade Portuguesa de Alergologia e Imunologia Clínica. [consultado 2020 jan 12]. Disponível em: https://www.spaic.pt/projectos/aria.

37. Bousquet J, Bedbrook A, Czarlewski W, Onorato GL, Arnavielhe S, Laune D, et al. Guidance to 2018 good practice: ARIA digitally-enabled, integrated, person-centred care for rhinitis and asthma. Clin Trans Allergy. 2019;9:16

38. Bédard A, Basagaña X, Anto JM, Garcia-Aymerich J, Devillier $P$, Arnavielhe $S$, et al. Mobile technology offers novel insights into the control and treatment of allergic rhinitis: The MASK study. J Allergy Clin Immunol. 2019;144:135-43.e6.

39. International Primary Care Respiratory Group. 9 $9^{\text {th }}$ IPCRG World Conference Abstract Book. Edinburgo: IPCRG; 2018

40. Bousquet J, Agache I, Aliberti MR, Angles R, Annesi-Maesano I, Anto JM, et al. Transfer of innovation on allergic rhinitis and asthma multimorbidity in the elderly (MACVIA-ARIA) - EIP on AHA Twinning Reference Site (GARD research demonstration project). Allergy. 2018;73:77-92.

41. Meltzer EO, Wallace D, Dykewicz M, Shneyer L. Minimal Clinically Important Difference (MCID) in allergic rhinitis: agency for healthcare research and quality or anchor-based thresholds? J Allergy Clin Immunol Pract. 2016;4:682-8.e6.

42. Sherman RE, Anderson SA, Dal Pan GJ, Gray GW, Gross T, Hunter NL et al. Real-world evidence - what is it and what can it tell us? N Engl J Med. 2016;375:2293-7.

43. Brozek JL, Bousquet J, Baena-Cagnani CE, Bonini S, Canonica GW, Casale TB, et al. Allergic Rhinitis and its Impact on Asthma (ARIA) guidelines: 2010 revision. J Allergy Clin Immunol. 2010;126:466-76.

44. Bousquet J, Schünemann HJ, Togias A, Bachert C, Erhola M, Hellings PW, et al. Next-generation Allergic Rhinitis and Its Impact on Asthma (ARIA) guidelines for allergic rhinitis based on Grading of Recommendations Assessment, Development and Evaluation (GRADE) and real-world evidence. J Allergy Clin Immunol. 2020;145:70-80.e3.

45. Dykewicz MS, Wallace DV, Baroody F, Bernstein J, Craig T, Finegold I, et al. Treatment of seasonal allergic rhinitis: an evidence-based focused 2017 guideline update. Ann Allergy Asthma Immunol. 2017;119:489 511.e41.

46. Bousquet J, Devillier P, Anto JM, Bewick M, Haahtela T, Arnavielhe S, et al. Daily allergic multimorbidity in rhinitis using mobile technology: a novel concept of the MASK study. Allergy. 2018;73:1622-31.

47. Bousquet J, Devillier P, Arnavielhe S, Bedbrook A, Alexis-Alexandre G, van Eerd $\mathrm{M}$, et al. Treatment of allergic rhinitis using mobile technology with real-world data: the MASK Observational Pilot Study. Allergy. 2018;73:1763-74.

48. Bousquet J, Schünemann HJ, Hellings PW, Arnavielhe S, Bachert C, Bedbrook A, et al. MACVIA clinical decision algorithm in adolescents and adults with allergic rhinitis. J Allergy Clin Immunol. 2016;138:36774.e2.

49. Courbis AL, Murray RB, Arnavielhe S, Caimmi D, Bedbrook A, Van Eerd $M$, et al. Electronic clinical decision support system for allergic rhinitis management: MASK e-CDSS. Clin Exp Allergy. 2018;48:1640-53.

50. Horak F, Bruttmann G, Pedrali P, Weeke B, Frølund L, Wolff HH, et al. A multicentric study of loratadine, terfenadine and placebo in patients with seasonal allergic rhinitis. Arzneimittelforschung. 1988;38:124-8.

51. Hampel FC, Ratner PH, Van Bavel J, Amar NJ, Daftary P, Wheeler W, et al. Double-blind, placebo-controlled study of azelastine and fluticasone in a single nasal spray delivery device. Ann Allergy Asthma Immunol. 2010;105:168-73.

52. Kaszuba SM, Baroody FM, deTineo M, Haney L, Blair C, Naclerio RM. Superiority of an intranasal corticosteroid compared with an oral antihistamine in the as-needed treatment of seasonal allergic rhinitis. Arch Intern Med. 2001;161:2581-7.

53. Glacy J, Putnam K, Godfrey S, Falzon L, Mauger B, Samson D, et al. Treatments for seasonal allergic rhinitis. Rockville: Agency for Healthcare Research and Quality; 2013.

55. U.S. Department of Health and Human Services Food and Drug Administration Center for Drug Evaluation and Research (CDER) Allergic rhinitis: developping drug products for treatment. Guidance for industry. 2016. [consultado 2020 jan 12]. Disponível em: https://www. fda.gov/downloads/drugs/guidances/ucm071293.pdf.

56. Katial RK, Salapatek AM, Patel P. Establishing the onset of action of intranasal corticosteroids: is there an ideal study design? Allergy Asthma 
Proc. 2009;30:595-604.

57. Patel P, D'Andrea C, Sacks HJ. Onset of action of azelastine nasal spray compared with mometasone nasal spray and placebo in subjects with seasonal allergic rhinitis evaluated in an environmental exposure chamber. Am J Rhinol. 2007;21:499-503.

58. Bousquet J, Meltzer EO, Couroux P, Koltun A, Kopietz F, Munzel U, et al. Onset of action of the fixed combination intranasal azelastinefluticasone propionate in an allergen exposure chamber. J Allergy Clin Immunol Pract. 2018;6:1726-32.e6.

59. Patel P, Roland PS, Marple BF, Benninger PJ, Margalias H, Brubaker M, et al. An assessment of the onset and duration of action of olopatadine nasal spray. Otolaryngol Head Neck Surg. 2007;137:918-24.

60. Salapatek AM, Lee J, Patel D, D'Angelo P, Liu J, Zimmerer RO Jr, et al. Solubilized nasal steroid (CDX-947) when combined in the same solution nasal spray with an antihistamine (CDX-313) provides improved, fastacting symptom relief in patients with allergic rhinitis. Allergy Asthma Proc. 2011;32:221-9.

61. Horak F, Zieglmayer UP, Zieglmayer R, Kavina A, Marschall K, Munzel $U$, et al. Azelastine nasal spray and desloratadine tablets in polleninduced seasonal allergic rhinitis: a pharmacodynamic study of onset of action and efficacy. Curr Med Res Opin. 2006;22:151-7.

62. Zieglmayer P, Zieglmayer R, Bareille P, Rousell V, Salmon E, Horak F. Fluticasone furoate versus placebo in symptoms of grass-pollen allergic rhinitis induced by exposure in the Vienna Challenge Chamber. Curr Med Res Opin. 2008;24:1833-40.

63. Murdoch RD, Bareille P, Ignar D, Miller SR, Gupta A, Boardley R, et al. The improved efficacy of a fixed-dose combination of fluticasone furoate and levocabastine relative to the individual components in the treatment of allergic rhinitis. Clin Exp Allergy. 2015;45:1346-355.

64. Sleurs K, Seys SF, Bousquet J, Fokkens WJ, Gorris S, Pugin B, et al. Mobile health tools for the management of chronic respiratory diseases. Allergy. 2019;74:1292-306.

65. Menditto E, Guerriero F, Orlando V, Crola C, Di Somma C, Illario M, et al. Self-assessment of adherence to medication: a case study in Campania region community-dwelling population. J Aging Res. 2015;2015:682503.

66. Bonini M. Electronic health (e-Health): emerging role in asthma. Curr Opin Pulm Med. 2017;23:21-6.

67. Pizzulli A, Perna S, Florack J, Pizzulli A, Giordani P, Tripodi S, et al. The impact of telemonitoring on adherence to nasal corticosteroid treatment in children with seasonal allergic rhinoconjunctivitis. Clin Exp Allergy. 2014;44:1246-54.

68. Ben Freedman S, Gersh B, Lip G. Misperceptions of aspirin efficacy and safety may perpetuate anticoagulant underutilization in atrial fibrillation. Eur Heart J. 2015;36:653-6.

69. Price D, Scadding G, Ryan D, Bachert C, Canonica GW, Mullol J, et al. The hidden burden of adult allergic rhinitis: UK healthcare resource utilisation survey. Clin Transl Allergy. 2015;5:39.

70. Bousquet J, Murray R, Price D, Somekh D, Münter L, Phillips J, et al. The allergic allergist behaves like a patient. Ann Allergy Asthma Immunol. 2018;121;741-2.

71. Patel P, Patel D, Kunjibettu S, Hall N, Wingertzahn MA. Onset of action of ciclesonide once daily in the treatment of seasonal allergic rhinitis. Ear Nose Throat J. 2008;87:340-53.

72. Wallace DV, Dykewicz MS, Bernstein DI, Blessing-Moore J, Cox L, Khan DA, et al. The diagnosis and management of rhinitis: an updated practice parameter. J Allergy Clin Immunol. 2008;122:S1-84.

73. Roberts G, Xatzipsalti M, Borrego LM, Custovic A, Halken S, Hellings PW, et al. Paediatric rhinitis: position paper of the European Academy of Allergy and Clinical Immunology. Allergy. 2013;68:1102-16.

74. Scadding GK, Kariyawasam HH, Scadding G, Mirakian R, Buckley RJ, Dixon $\mathrm{T}$, et al. BSACl guideline for the diagnosis and management of allergic and non-allergic rhinitis. Clin Exp Allergy. 2017;47:856-89.

75. Carr W, Bernstein J, Lieberman P, Meltzer E, Bachert C, Price D, et al. A novel intranasal therapy of azelastine with fluticasone for the treatment of allergic rhinitis. J Allergy Clin Immunol. 2012;129:1282-9.e10.

76. Meltzer EO. Pharmacotherapeutic strategies for allergic rhinitis: matching treatment to symptoms, disease progression, and associated conditions. Allergy Asthma Proc. 2013;34:301-11.

77. Seidman MD, Gurgel RK, Lin SY, Schwartz SR, Baroody FM, Bonner JR, et al. Clinical practice guideline: allergic rhinitis executive summary. Otolaryngol Head Neck Surg. 2015;152:197-206

78. Seidman MD, Gurgel RK, Lin SY, Schwartz SR, Baroody FM, Bonner $\mathrm{JR}$, et al. Clinical practice guideline: allergic rhinitis. Otolaryngol Head Neck Surg. 2015;152:S1-43.

79. Bachert C, Bousquet J, Hellings P. Rapid onset of action and reduced nasal hyperreactivity: new targets in allergic rhinitis management. Clin
Transl Allergy. 2018:8:25.

80. Church M, Maurer M, Simons F, Bindslev-Jensen C, van Cauwenberge $\mathrm{P}$, Bousquet J, et al. Risk of first-generation H(1)-antihistamines: a GA(2) LEN position paper. Allergy. 2010;65:459-66.

81. Halken S, Larenas-Linnemann D, Roberts G, Calderón MA, Angier E, Pfaar $\mathrm{O}$, et al. EAACI guidelines on allergen immunotherapy: prevention of allergy. Pediatr Allergy Immunol. 2017;28:728-45.

82. Bonertz A, Roberts G, Slater JE, Bridgewater J, Rabin RL, Hoefnagel $M$, et al. Allergen manufacturing and quality aspects for allergen immunotherapy in Europe and the United States: an analysis from the EAACI AIT Guidelines Project. Allergy. 2018;73:816-26.

83. Roberts G, Pfaar O, Akdis CA, Ansotegui IJ, Durham SR, Gerth van Wijk R, et al. EAACI Guidelines on Allergen Immunotherapy: Allergic Rhinoconjunctivitis. Allergy. 2018;73:765-98.

84. Ryan D, van Wijk RG, Angier E, Kristiansen M, Zaman H, Sheikh A, et al. Challenges in the implementation of the EAACI AIT guidelines: a situational analysis of current provision of allergen immunotherapy. Allergy. 2018;73:827-36.

85. Pfaar O, Bachert C, Bufe A, Buhl R, Ebner C, Eng P, et al. Guideline on allergen-specific immunotherapy in IgE-mediated allergic diseases: S2k Guideline of the German Society for Allergology and Clinical Immunology (DGAKI), the Society for Pediatric Allergy and Environmental Medicine (GPA), the Medical Association of German Allergologists (AeDA), the Austrian Society for Allergy and Immunology (ÖGAI), the Swiss Society for Allergy and Immunology (SGAI), the German Society of Dermatology (DDG), the German Society of Oto- Rhino-Laryngology, Head and Neck Surgery (DGHNO-KHC), the German Society of Pediatrics and Adolescent Medicine (DGKJ), the Society for Pediatric Pneumology (GPP), the German Respiratory Society (DGP), the German Association of ENT Surgeons (BV-HNO), the Professional Federation of Paediatricians and Youth Doctors (BVKJ), the Federal Association of Pulmonologists (BDP) and the German Dermatologists Association (BVDD). Allergo J. 2014;23:282-319.

86. Muraro A, Roberts G, Halken S, Agache I, Angier E, Fernandez-Rivas $\mathrm{M}$, et al. EAACl guidelines on allergen immunotherapy: executive statement. Allergy. 2018;73:739-43.

87. Larenas-Linnemann DE, Antolín-Amérigo D, Parisi C, Nakonechna A, Luna-Pech JA, Wedi B, et al. National clinical practice guidelines for allergen immunotherapy: an international assessment applying AGREEII. Allergy. 2018;73:664-72.

88. Zielen S, Devillier P, Heinrich J, Richter H, Wahn U. Sublingual immunotherapy provides long-term relief in allergic rhinitis and reduces the risk of asthma: a retrospective, real-world database analysis. Allergy. 2018;73:165-77.

89. Devlin N, Parkin D. Does NICE have a cost-effectiveness threshold and what other factors influence its decisions? A binary choice analysis. Health Econ. 2004;13:437-52.

90. Meadows A, Kaambwa B, Novielli N, Huissoon A, Fry-Smith A, Meads C, et al. A systematic review and economic evaluation of subcutaneous and sublingual allergen immunotherapy in adults and children with seasonal allergic rhinitis. Health Technol Assess. 2013;17:1-322.

91. Bousquet J, Pfaar O, Togias A, Schünemann HJ, Ansotegui I, Papadopoulos NG, et al. 2019 ARIA care pathways for allergen immunotherapy. Allergy. 2019;74:2087-102.

92. Bonertz A, Roberts GC, Hoefnagel M, Timon M, Slater JE, Rabin RL, et al. Challenges in the implementation of EAACl guidelines on allergen immunotherapy: a global perspective on the regulation of allergen products. Allergy. 2018;73:64-76.

93. Kowalski ML, Ansotegui I, Aberer W, Al-Ahmad M, Akdis M, BallmerWeber BK, et al. Risk and safety requirements for diagnostic and therapeutic procedures in allergology: World Allergy Organization Statement. World Allergy Organ J. 2016;9:33.

94. Bachert C, Larché M, Bonini S, Canonica GW, Kündig T, LarenasLinnemann $D$, et al. Allergen immunotherapy on the way to productbased evaluation-a WAO statement. World Allergy Organ J. 2015;8:29.

95. Committee for Medicinal Products for Human Use (CPMP). Guideline on allergen products: production and quality issues. Londres: European Medicines Agency; 2008.

96. Cox L, Larenas-Linnemann D, Lockey RF, Passalacqua G. Speaking the same language: The World Allergy Organization Subcutaneous Immunotherapy Systemic Reaction Grading System. J Allergy Clin Immunol. 2010;125:569-74, 574.e1-7.

97. Passalacqua G, Baena-Cagnani CE, Bousquet J, Canonica GW, Casale TB, Cox L, et al. Grading local side effects of sublingual immunotherapy for respiratory allergy: speaking the same language. J Allergy Clin Immunol. 2013;132:93-8. 
98. Baiardini I, Puggioni F, Menoni S, Boot JD, Diamant Z, Braido F, et al. Patient knowledge, perceptions, expectations and satisfaction on allergen-specific immunotherapy: a survey. Respir Med. 2013;107:3617.

99. Nam YH, Lee SK. Physician's recommendation and explanation is important in the initiation and maintenance of allergen immunotherapy. Patient Prefer Adherence. 2017;11:381-7.

100. Chivato T, Álvarez-Calderón P, Panizo C, Abengozar R, Alías C, AlBaech A, et al. Clinical management, expectations, and satisfaction of patients with moderate to severe allergic rhinoconjunctivitis treated with SQ-standardized grass-allergen tablet under routine clinical practice conditions in Spain. Clin Mol Allergy. 2017;15:1.

101. Skoner DP, Blaiss MS, Dykewicz MS, Smith N, Leatherman B, Bielory $\mathrm{L}$, et al. The Allergies, Immunotherapy, and RhinoconjunctivitiS (AIRS) survey: patients' experience with allergen immunotherapy. Allergy Asthma Proc. 2014;35:219-26.

102. Pitsios C, Dietis N. Ways to increase adherence to allergen immunotherapy. Curr Med Res Opin. 2019;35:1027-31.

103. Bender BG, Lockey RF. Solving the problem of nonadherence to immunotherapy. Immunol Allergy Clin North Am. 2016;36:205-13.

104. Kuehl BL, Abdulnour S, O'Dell M, Kyle TK. Understanding the role of the healthcare professional in patient self-management of allergic rhinitis. SAGE Open Med. 2015;3: 2050312115595822.

105. Bosnic-Anticevich S, Kritikos V, Carter V, Yan KY, Armour C, Ryan D, et al. Lack of asthma and rhinitis control in general practitioner-managed patients prescribed fixed-dose combination therapy in Australia. J Asthma. 2018;55:684-94.

106. Bosnic-Anticevich S, Costa E, Menditto E, Lourenço O, Novellino E, Bialek S, et al. ARIA pharmacy 2018 "Allergic rhinitis care pathways for community pharmacy": AIRWAYS ICPs initiative (European Innovation Partnership on Active and Healthy Ageing, DG CONNECT and DG Santé) POLLAR (Impact of Air POLLution on Asthma and Rhinitis) GARD Demonstration project. Allergy. 2019;74:1219-36.

107. INFARMED. Normas relativas à dispensa de medicamentos e produtos de saúde. 2019 [consultado 2020 jan 12]. Disponível em: https://www. infarmed.pt/documents/15786/17838/Normas_Dispensa/4c1aea02a266-4176-b3ee-a2983bdfe790.

108. INFARMED. Normas relativas à prescrição de medicamentos e produtos de saúde. 2019 [consultado 2020 jan 12]. Disponível em: https://www.infarmed.pt/documents/15786/17838/Normas Prescri\%C3\%A7\%C3\%A3o/bcd0b378-3b00-4 ee 0-910428d0db0b7872.

109. Jutel M, Papadopoulos NG, Gronlund H, Hoffman HJ, Bohle B, Hellings $\mathrm{P}$, et al. Recommendations for the allergy management in the primary care. Allergy. 2014;69:708-18.

110. Finlay I, Egner W. Allergy - will we ever meet the unmet need? J R Soc Med. 2010;103:430-1.

111. Hellings PW, Fokkens WJ, Bachert C, Akdis CA, Bieber T, Agache I, et al. Positioning the principles of precision medicine in care pathways for allergic rhinitis and chronic rhinosinusitis - A EUFOREA-ARIA-EPOSAIRWAYS ICP statement. Allergy. 2017;72:1297-305.

112. Pinnock H, Thomas M, Tsiligianni I, Lisspers K, Østrem A, Ställberg $B$, et al. The International Primary Care Respiratory Group (IPCRG) Research Needs Statement 2010. Prim Care Respir J. 2010;19:S1-20.

113. Shehata Y, Ross M, Sheikh A. Undergraduate allergy teaching in a UK medical school: comparison of the described and delivered curriculum. Prim Care Respir J. 2007;16:16-21.

114. Alvarez-Cuesta E, Bousquet J, Canonica GW, Durham SR, Malling HJ, Valovirta E, et al. Standards for practical allergen-specific immunotherapy. Allergy. 2006;61:S1-20.

115. Landi M, Meglio P, Praitano E, Lombardi C, Passalacqua G, Canonica GW. The perception of allergen-specific immunotherapy among pediatricians in the primary care setting. Clin Mol Allergy. 2015;13:15.
116. Lockey RF, Hankin CS. Health economics of allergen-specific immunotherapy in the United States. J Allergy Clin Immunol. 2011;127:39-43.

117. Asaria M, Dhami S, van Ree R, Gerth van Wijk R, Muraro A, Roberts $G$, et al. Health economic analysis of allergen immunotherapy for the management of allergic rhinitis, asthma, food allergy and venom allergy: a systematic overview. Allergy. 2018;73:269-83.

118. Canonica GW, Bachert C, Hellings P, Ryan D, Valovirta E, Wickman M, et al. Allergen Immunotherapy (AIT): a prototype of precision medicine World Allergy Organ J. 2015;8:31.

119. Boni E, Incorvaia C, Makrì E, Preziosi D, Mauro M. When allergen immunotherapy perfectly meets its need: a case report. J Allergy Ther 2017;08:2.

120. Canonica GW, Ansotegui IJ, Pawankar R, Schmid-Grendelmeier P, van Hage M, Baena-Cagnani CE, et al. A WAO - ARIA - GA²LEN consensus document on molecular-based allergy diagnostics. World Allergy Organ J. 2013;6:17

121. O'Hehir RE, Varese NP, Deckert K, Zubrinich CM, van Zelm MC, Rolland $\mathrm{JM}$, et al. Epidemic thunderstorm asthma protection with five-grass pollen tablet sublingual immunotherapy: a clinical trial. Am J Respir Crit Care Med. 2018;198:126-8.

122. Lee J, Kronborg C, O'Hehir RE, Hew M. Who's at risk of thunderstorm asthma? The ryegrass pollen trifecta and lessons learnt from the Melbourne thunderstorm epidemic. Respiratory Medicine. 2017;132:146-8

123. Amaral R, Fonseca JA, Jacinto T, Pereira AM, Malinovschi A, Janson $C$, et al. Having concomitant asthma phenotypes is common and independently relates to poor lung function in NHANES 2007-2012. Clin Transl Allergy. 2018;8:13.

124. European Medicines Agency. Summary of product characteristics. Acarizax 12 SQ-HDM oral lyophilisate. In: Summary of product characteristics. Acarizax 12 SQ-HDM oral lyophilisate . 2016 [consultado 2020 jan 12]. Disponível em: https://mricts-mrp.eu/Human/Downloads/ DE H 1947001 FinalSPC.pdf.

125. Masuyama K, Okamoto Y, Okamiya K, Azuma R, Fujinami T, Riis B, et al. Efficacy and safety of SQ house dust mite sublingual immunotherapytablet in Japanese children. Allergy. 2018;73:2352-63.

126. Penagos M, Eifan AO, Durham SR, Scadding GW. Duration of allergen immunotherapy for long-term efficacy in allergic rhinoconjunctivitis. Curr Treat Options Allergy. 2018;5:275-90.

127. Valovirta E, Petersen TH, Piotrowska T, Laursen MK, Andersen JS, Sørensen HF, et al. Results from the 5-year $\mathrm{SQ}$ grass sublingual immunotherapy tablet asthma prevention (GAP) trial in children with grass pollen allergy. J Allergy Clin Immunol. 2018;141:529-38.e13.

128. Möller C, Dreborg S, Ferdousi HA, Halken S, Høst A, Jacobsen L, et al. Pollen immunotherapy reduces the development of asthma in children with seasonal rhinoconjunctivitis (the PAT-study). J Allergy Clin Immunol. 2002;109:251-6

129. Kristiansen M, Dhami S, Netuveli G, Halken S, Muraro A, Roberts G, et al. Allergen immunotherapy for the prevention of allergy: A systematic review and meta-analysis. Pediatr Allergy Immunol. 2017;28:18-29.

130.Bożek A, Kołodziejczyk K, Kozłowska R, Canonica GW. Evidence of the efficacy and safety of house dust mite subcutaneous immunotherapy in elderly allergic rhinitis patients: a randomized, double-blind placebocontrolled trial. Clin Transl Allergy. 2017;7:43

131. Bousquet J, Arnavielhe S, Bedbrook A, Fonseca J, Morais Almeida M, Todo Bom A, et al. The Allergic Rhinitis and its Impact on Asthma (ARIA) score of allergic rhinitis using mobile technology correlates with quality of life: the MASK Study. Allergy. 2018;73:505-10.

132. Pfaar O, Demoly P, Gerth van Wijk R, Bonini S, Bousquet J, Canonica $\mathrm{GW}$, et al. Recommendations for the standardization of clinical outcomes used in allergen immunotherapy trials for allergic rhinoconjunctivitis: an EAACI Position Paper. Allergy. 2014;69:854-67. 